\title{
Characteristics of non-Darcy flow in low-permeability reservoirs
}

\author{
Yao Yuedong* and Ge Jiali \\ Key Laboratory of Petroleum Engineering of the Ministry of Education, China University of Petroleum, Beijing 102249, \\ China
}

(C) China University of Petroleum (Beijing) and Springer-Verlag Berlin Heidelberg 2011

\begin{abstract}
Well testing is recognized as an effective means of accurately obtaining the formation parameters of low-permeability reservoirs and effectively analyzing the deliverability. Well test models must comply with the particular characteristics of flow in low-permeability reservoirs in order to obtain reasonable well test interpretation. At present, non-Darcy flow in low-permeability reservoirs is attracting much attention. In this study, displacement tests were conducted on typical cores taken from low-permeability reservoirs. Two dimensionless variables were introduced to analyze the collected experimental data. The results of the dimensionless analysis show whether non-Darcy flow happens or not depends on the properties of fluid and porous media and the pressure differential. The combination of the above three parameters was named as dimensionless criteria coefficient (DCC). When the value of the DCC was lower than a critical Reynolds number (CRN), the flow could not be well described by Darcy's law (so-called non-Darcy flow), when the DCC was higher than CRN, the flow obeyed Darcy's law. Finally, this paper establishes a transient mathematical model considering Darcy flow and nonDarcy flow in low-permeability reservoirs, and proposes a methodology to solve the model. The solution technique, which is based on the Boltzmann transformation, is well suited for solving the flow model of low-permeability reservoirs. Based on the typical curves analysis, it was found that the pressure and its derivative curves were determined by such parameters as non-Darcy flow index and the flow characteristics. The results can be used for well test analysis of low-permeability reservoirs.
\end{abstract}

Key words: Low-permeability, non-Darcy flow, dimensionless analysis, transient flow, well testing

\section{Introduction}

Low-permeability reservoirs form one of the important resources in China according to the statistics of Jiang et al (2004). Low-permeability reservoirs have different characteristics from the conventional ones. Non-Darcy flow occurs in the low-permeability reservoirs (Yan et al, 1990). When the pressure gradient $\Delta p$ is relatively low, the fluid flow in low-permeability reservoirs does not obey Darcy's law, i.e. the fluid velocity $v$ is not proportional to the pressure gradient; when the pressure gradient is relatively high, the fluid velocity increases linearly with the pressure gradient (Huang, 1998). Extrapolating the straight part of the $\Delta p-v$ curve to the pressure gradient axis, the pseudo threshold pressure gradient is obtained. The equation including the threshold pressure gradient was firstly used to describe fluid flow in low-permeability reservoirs. To increase the prediction accuracy of the flow equation, a three-parameter model was presented (Deng and Liu, 2001; Deng et al, 2007; 2009). Later, the model was simplified to a two-parameter model

*Corresponding author. email: yaoyuedong@163.com

Received January 15, 2010
(Yang, 2007). The threshold pressure gradient and other parameters mentioned in these models were only determined by specific core tests. This makes the model difficult to apply to general cases.

Ren et al (2009) proposed a new Non-Darcy flow equation for single-phase flow (oil or water) in lowpermeability porous media. This equation took into account the pore structure of reservoir rocks, fluid properties, and the pressure gradient. However, this equation is not applicable to low-velocity fluid flow in porous media. For example, the flow rate calculated from this equation is not zero when the pressure gradient is zero, which contradicts the flow characteristics in actual reservoir formations. Based on the core flow tests, Yao and Ge (2001) concluded that the flow within the low-permeability cores was non-linear when the dimensionless criteria coefficient (DCC) was lower than the critical Reynolds number (CRN, $\left.8.5 \times 10^{-5}\right)$; and the flow was linear when it exceeded CRN. The experimental results were also in agreement with microscopic boundary layer theory. In order to achieve more satisfactory results, the resistance coefficient of the above model was modified by Liu and Liu (2003). However, the new resistance coefficient is not easy to apply to general cases due to varying threshold pressure 
gradient in different cores. Using the method proposed by Yao and Ge in 2001, Li et al (2005) drew the same conclusion except for a slightly difference in the critical Reynolds number $\left(R e_{\mathrm{c}}=8.95 \times 10^{-5}\right)$.

For non-Darcy transient flow in low-permeability reservoirs, it is convenient to choose the Izbash equation (Bordier and Zimmer, 2000) to describe non-Darcy flow. The Izbash equation indicates that the hydraulic gradient is a power function of the flow rate. Izbash's law has been preferred to derive drainage equations because it is in continuity with Darcy's law and facilitates the development of an analytical solution. Because of its nonlinearity, the Izbash equation is usually simplified by the linear approximation, and then an approximate solution is obtained (Ikoku and Ramey, 1979). The above approximation is applicable only for fluid flow near the borehole or in large time, and the radial flow rate calculated is only accurate in the wellbore. Assuming that the flow rate is constant at any time and any space, the longer the distance from the wellbore and the earlier the time is, the greater the error is. The same approximate linear method was applied to study non-Newton fluid flow (Ikoku and Ramey, 1982; Tong and Shi, 2004; Tong and Wang, 2004; Li et al, 2007; Zhang and Yue, 2007). Other researchers studied the transient flow in low-permeability reservoirs using the nonDarcy flow model with the additional threshold pressure gradient (Liu, 1982; Cheng et al, 1996; Song and Liu,1999; Hou and Tong, 2009; Xiong et al, 2009), while the above method is restricted to set the exact value of the threshold pressure gradient just as mentioned above. By using the power function of the Izbash equation (Wen et al, 2008), a single power function was used in the equation of the whole flow regime. The above method should divide into regimes individually according to the distribution of flow pattern. The dimensionless bottom-hole pressure solution of above model is lower than Darcy's flow, it is obviously unreasonable.

In this study, we conducted a number of core tests on lowpermeability reservoir rocks to determine the flow equation and critical Reynolds number for non-Darcy flow in lowpermeability reservoirs. According to the characteristics of fluid flow in low-permeability reservoirs, we developed a complex model for transient flow in low-permeability reservoirs, solved the flow model considering the variation of parameters such as the distribution of flow pattern and seepage index, and then analyzed the transient pressure and its influencing factors.

\section{Flow tests on low-permeability cores}

\subsection{Cores and flowing medium}

Flow tests were performed on 28 cylindrical core samples taken from low-permeability reservoirs. The core plugs were $8 \mathrm{~cm}$ long and $2.5 \mathrm{~cm}$ in diameter. The gas permeability and porosity of these samples ranged from 0.017 to $50 \times 10^{-3} \mu \mathrm{m}^{2}$ and 0.095 to 0.218 , respectively. There were no structural defects like surface cracks, bedding planes, and corrosion holes etc. All samples were consistent with the characteristics of a sedimentary environment. The fluids used in core flow tests were $8 \% \mathrm{KCl}$ solution, kerosene, and simulated oils A and B. Simulated oils A and B were prepared by blending degassed oil with kerosene in a ratio of $1: 1$ and $1: 3$, respectively. The physical properties of these fluids were listed in Table 1.

Table 1 Physical properties of fluids used in flow tests

\begin{tabular}{ccc}
\hline Fluid & Relative density & Viscosity, $\mathrm{mPa} \cdot \mathrm{s}$ \\
\hline $8 \% \mathrm{KCl}$ solution & 1.01 & 0.89 \\
Kerosene & 0.80 & 0.90 \\
Simulated oil A & 0.84 & 5.46 \\
Simulated oil B & 0.82 & 2.13 \\
\hline
\end{tabular}

\subsection{Experimental equipment and method}

The experimental equipment was mainly composed of a flowing system, a pressure control system, and a data acquisition system, as shown in Fig. 1. Core flooding tests were conducted in a conventional manner at room temperature $\left(20^{\circ} \mathrm{C}\right)$. The core was saturated with the fluid of known density and viscosity.

The pump connected to the experimental fluid container maintained the desired flow rate through the core. The outlet of the pump was connected to the core. The effluents from the core outlet were collected in a fraction collector. A differential

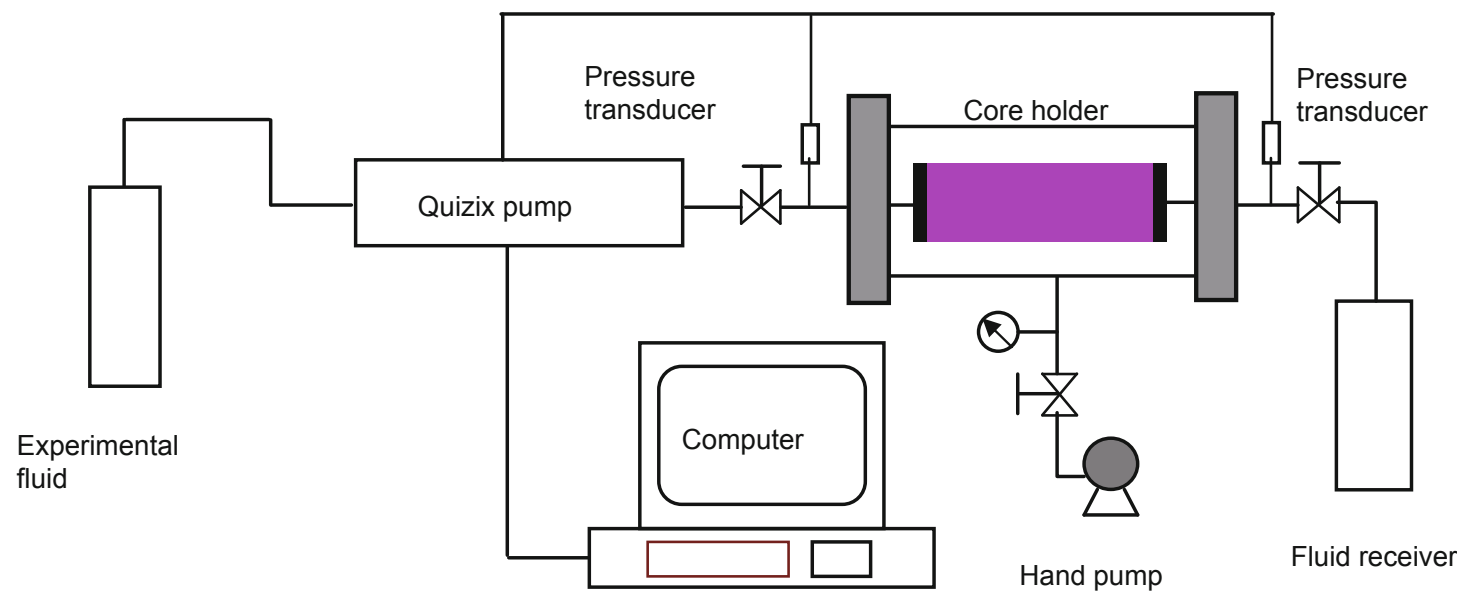

Fig. 1 A schematic diagram of the flow test apparatus 
pressure transmitter was connected between the inlet and outlet of the core to measure the pressure difference across the core.

A back pressure pump was used to pump fluid through the core sample at a designed inlet pressure, and a receiving pump was used at the other end of the sample to control the outlet pressure. The flow rate was read from the pump. Tests should be repeated several times at low flow rates and relatively high flow rates to ensure that the Darcy and nonDarcy flow behavior were taking place. A plot of pressure gradient versus flow rate would give a straight line in the Darcy regime and a deviated upward trend in the non-Darcy regime.

\subsection{Experimental results and analysis}

Based on all collected data of pressure gradient versus flow rate, set

$$
\begin{aligned}
& f=\delta \frac{\Delta p}{\rho \Delta l}\left(\frac{\phi A}{q}\right)^{2} \\
& R e=\frac{q \rho \delta}{\mu A \phi}
\end{aligned}
$$

then, a plot of dimensionless groups $f$ versus $R e$ was been given (Fig. 2).

As for the curve trend of Fig. 2, the slope of curve

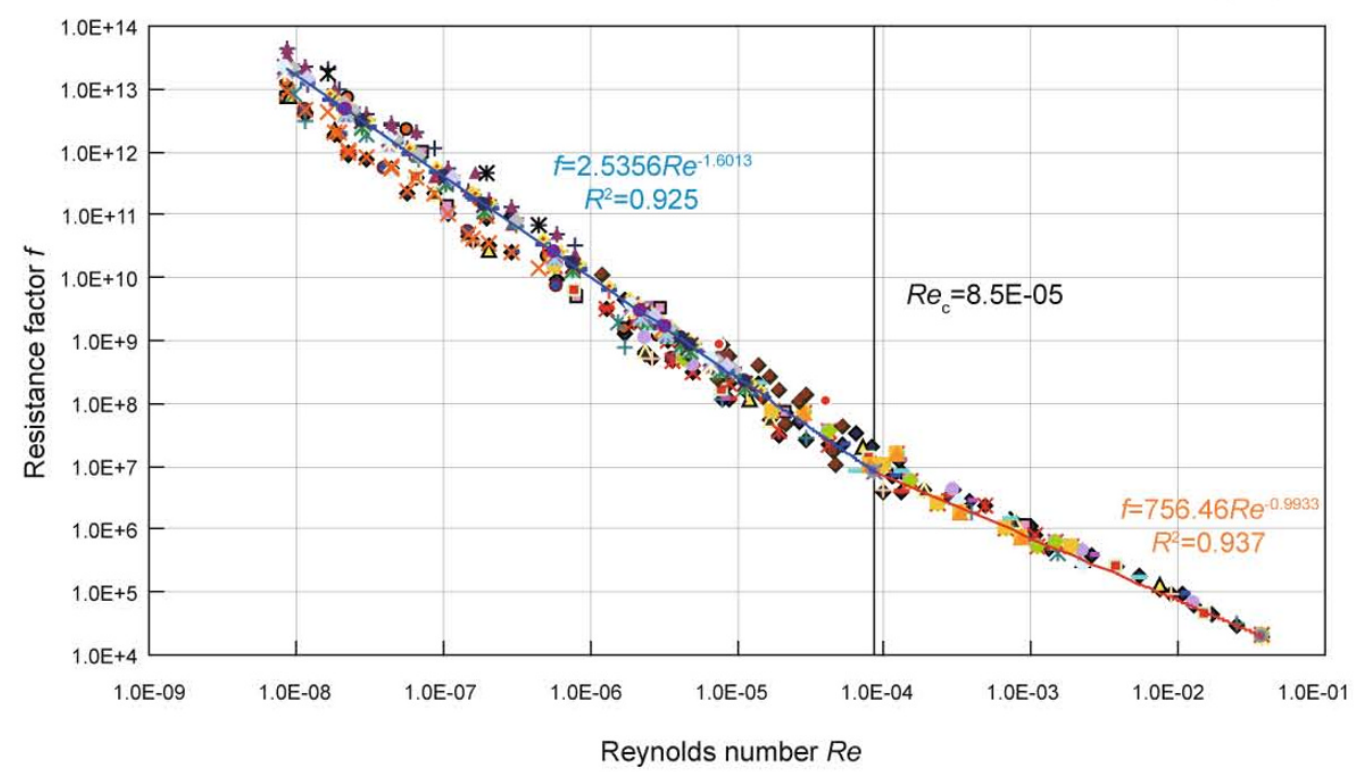

Fig. 2 The dimensionless experimental curve of flow in low-permeability cores

increased as the $R e$ decreases, but the whole curve could be divided into two straight lines approximately, the critical point was: $R e_{\mathrm{c}}=8.5 \times 10^{\mathrm{fi}} 5$.

\subsubsection{The left straight line}

The left straight line is the best fit of the data, with a correlation coefficient $R^{2}$ of 0.925 . The fitted equation is:

$$
\lg f=\lg 2.5356-1.6013 \lg R e
$$

Then

$$
\delta \frac{\Delta p}{\rho \Delta l}\left(\frac{\phi A}{q}\right)^{2}\left(\frac{q \rho \delta}{\mu A \phi}\right)^{1.6013}=2.5356
$$

So

$$
\frac{\Delta p}{\Delta l}=c_{1} v^{0.3987}
$$

The above is non-Darcy flow equation.

\subsubsection{The right straight line}

Similarly, the right straight line $f=756.46 R e^{-0.9933}$ $\left(R^{2}=0.937\right)$ is the best fit to the data when $R \mathrm{e}>8.5 \times 10^{\mathrm{fi} 5}$.

So

$\frac{\Delta p}{\Delta l}=c_{2} v$
The above is Darcy flow equation, in which $\delta$ is the characteristic scale of porous media, $\mathrm{m} ; \Delta p$ is the difference between the inlet and outlet pressures, $\mathrm{MPa} ; \rho$ is the density, $\mathrm{kg} / \mathrm{m}^{3} ; \Delta L$ is the length, $\mathrm{m} ; \mu$ is the porosity of the medium, fraction; $A$ is the cross area of core, $\mathrm{m}^{2} ; q$ is the surface flow rate, $\mathrm{m}^{3} / \mathrm{s} ; \mu$ is the viscosity, $\mathrm{mPa} \cdot \mathrm{s} ; C_{1}$ and $C_{2}$ are constant.

\section{Continuity equation for non-Darcy flow in low-permeability reservoirs}

\subsection{Continuity equation for radial flow}

For an infinitesimal circular element, as shown in Fig. 3, the quantity of fluid that will flow into the element over a time interval $\mathrm{d} t$ is:

$$
\left[\rho v_{r}-\frac{\partial\left(\rho v_{r}\right)}{\partial r} \frac{\mathrm{d} r}{2}\right] \times 2 \pi\left(r-\frac{\mathrm{d} r}{2}\right) \mathrm{d} z \mathrm{~d} t
$$

For the radial fluid flow, the element above has no connection with the $z$ axis.

The quantity of fluid that will flow out the infinitesimal element over a time interval $\mathrm{d} t$ is: 


$$
\left[\rho v_{r}+\frac{\partial\left(\rho v_{r}\right)}{\partial r} \frac{\mathrm{d} r}{2}\right] \times 2 \pi\left(r+\frac{\mathrm{d} r}{2}\right) \mathrm{d} z \mathrm{~d} t
$$

Considering the compressibility of fluid and formation, the change of quantity of fluid over a time interval $\mathrm{d} t$ is:

$$
\frac{\partial}{\partial t}\left\{\rho \varphi\left[\pi(r+\mathrm{d} r / 2)^{2}-\pi(r-\mathrm{d} r / 2)^{2}\right]\right\} \mathrm{d} z \mathrm{~d} t
$$

According to the law of conservation of mass, the continuity equation can be expressed as:

$$
\frac{\partial\left(\rho v_{r}\right)}{\partial r}+\frac{1}{r} \rho v_{r}=-\frac{\partial(\rho \varphi)}{\partial t}
$$

For slightly compressible fluid flow, Eq. (8) can be simplified to

$$
\frac{\partial v(r, t)}{\partial r}+\frac{1}{r} v(r, t)=c_{\mathrm{t}} \frac{\partial p(r, t)}{\partial t}
$$

where $r$ is the radius distance, $\mathrm{m} ; k$ is the permeability, $\mathrm{mD} ; t$ is time, $\mathrm{s} ; c_{\mathrm{t}}$ is the system compressibility, $\mathrm{MPa}^{-1}$.

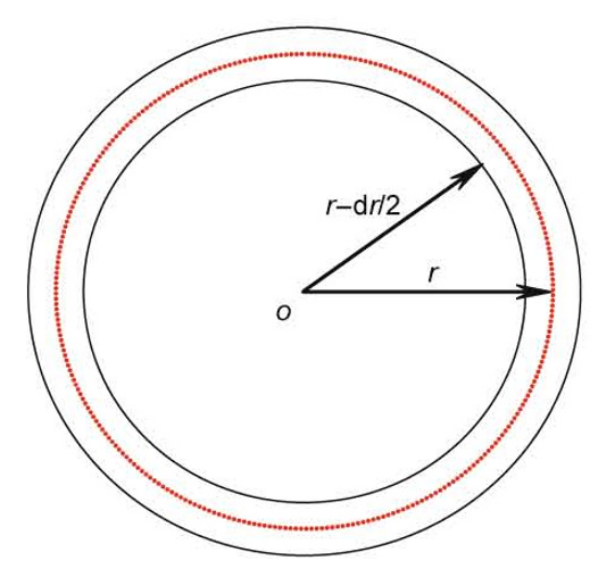

Fig. 3 The schematic of a circular element

\subsection{Continuity equation for non-Darcy flow}

The fluid flow in low-permeability reservoirs may be Darcy flow or non-Darcy flow, which means that the power law index $n$ for the non-Darcy flow may be a changing parameter, so it is necessary to do a multi-regional study according to different DCCs. For low-permeability reservoirs, low velocity non-Darcy flow usually happens in the area which is far away from the wellbore; utilizing a non-Darcy or Darcy flow model alone will not accurately describe fluid flow in the formation effectively. As a result, it is necessary to introduce a composite reservoir model considering both Darcy flow and non-Darcy flow. For an infinite reservoir producing at constant rate, the non-Darcy flow model can be expressed as follows:

$$
\left\{\begin{array}{lc}
\frac{\partial v(r, t)}{\partial r}+\frac{1}{r} v(r, t)=c_{\mathrm{t}} \frac{\partial p(r, t)}{\partial t} & \\
\frac{\partial p(r, t)}{\partial r}=c v_{\mathrm{p}}{ }^{n}(r, t) & 0<n<1, \quad r>r_{\mathrm{c}} \\
\frac{\partial p(r, t)}{\partial r}=\frac{\mu}{k} v_{\mathrm{d}}(r, t), \quad r_{\mathrm{w}} \leq r \leq r_{\mathrm{c}} \\
p(r, t=0)=p_{\mathrm{i}} & \text { Initial condition } \\
v_{\mathrm{d}}\left(r=r_{\mathrm{w}}, t\right)=q_{\mathrm{w}} / 2 \pi h r_{\mathrm{w}} & \text { Inner boundary condition } \\
v_{\mathrm{p}}\left(r=r_{\mathrm{c}}, t\right)=v_{\mathrm{d}}\left(r=r_{\mathrm{c}}, t\right) & \text { Continuous condition } \\
p(r=\infty, t)=p_{\mathrm{i}} & \text { Outer boundary condition }
\end{array}\right.
$$

The dimensionless variables are defined as follows:

$$
\begin{aligned}
& P_{\mathrm{D}}=\frac{k}{r_{\mathrm{w}} v_{\mathrm{w}} \mu}\left(p_{\mathrm{e}}-p\right) \\
& t_{\mathrm{D}}=\frac{k t}{\mu c_{\mathrm{t}} r_{\mathrm{w}}^{2}} \\
& r_{\mathrm{D}}=\frac{r}{r_{\mathrm{w}}} \\
& v_{\mathrm{D}}=\frac{v}{v_{\mathrm{w}}}
\end{aligned}
$$

Then Eq. (10) can be written as follows:

$$
\begin{aligned}
& \left\{\begin{array}{l}
\frac{\partial v_{\mathrm{D}}\left(r_{\mathrm{D}}, t_{\mathrm{D}}\right)}{\partial r_{\mathrm{D}}}+\frac{1}{r_{\mathrm{D}}} v_{\mathrm{D}}\left(r_{\mathrm{D}}, t_{\mathrm{D}}\right)=-\frac{\partial p_{\mathrm{D}}\left(r_{\mathrm{D}}, t_{\mathrm{D}}\right)}{\partial t_{\mathrm{D}}} \\
\frac{\partial p_{\mathrm{D}}\left(r_{\mathrm{D}}, t_{\mathrm{D}}\right)}{\partial r_{\mathrm{D}}}=-\frac{k v_{\mathrm{w}}^{n-1} c}{\mu} v_{\mathrm{pD}}^{n}\left(r_{\mathrm{D}}, t_{\mathrm{D}}\right) \quad 0<n<1, \quad r_{\mathrm{D}}>r_{\mathrm{cD}}
\end{array}\right. \\
& \left\{\frac{\partial p_{\mathrm{D}}\left(r_{\mathrm{D}}, t_{\mathrm{D}}\right)}{\partial r_{\mathrm{D}}}=-v_{\mathrm{dD}}\left(r_{\mathrm{D}}, t_{\mathrm{D}}\right) \quad 1 \leq r_{\mathrm{D}} \leq r_{\mathrm{cD}}\right. \\
& p_{\mathrm{D}}\left(r_{\mathrm{D}}, t_{\mathrm{D}}=0\right)=0 \quad \text { Initial condition } \\
& v_{\mathrm{dD}}\left(r_{\mathrm{D}}=1, t_{\mathrm{D}}\right)=1 \quad \text { Inner boundary condition } \\
& v_{\mathrm{pD}}\left(r_{\mathrm{D}}=r_{\mathrm{cD}}, t_{\mathrm{D}}\right)=v_{\mathrm{dD}}\left(r_{\mathrm{D}}=r_{\mathrm{cD}}, t_{\mathrm{D}}\right) \text { Continuous condition } \\
& p_{\mathrm{D}}\left(r_{\mathrm{D}}=\infty, t_{\mathrm{D}}\right)=0 \quad \text { Outer boundary condition }
\end{aligned}
$$

where $n$ is the seepage index; subscripts w denotes the well wall, D denotes dimensionless, $\mathrm{p}$ denotes regime of nonDarcy, d denotes regime of Darcy, and c denotes critical regime of Darcy; $p_{\mathrm{i}}$ is the initial reservoir pressure, $\mathrm{MPa}$. 


\section{Exact solutions to the non-Darcy flow model}

\subsection{The flow rate}

\subsubsection{Darcy flow regime $\left(1 \leq r_{\mathrm{D}} \leq r_{\mathrm{cD}}\right)$}

The Darcy flow equation is:

$$
\frac{\partial p_{\mathrm{D}}\left(r_{\mathrm{D}}, t_{\mathrm{D}}\right)}{\partial r_{\mathrm{D}}}=-v_{\mathrm{D}}\left(r_{\mathrm{D}}, t_{\mathrm{D}}\right)
$$

Let

$$
\eta=\frac{r_{\mathrm{D}}}{2 t_{\mathrm{D}}^{1 / 2}}
$$

Applying the Boltzmann transformation (Eq. (13)) to Eqs. (11-12) yields:

$$
\begin{aligned}
& \frac{\mathrm{d} v_{\mathrm{D}}(\eta)}{\mathrm{d} \eta}+\frac{1}{\eta} v_{\mathrm{D}}(\eta)=\frac{\eta}{t_{\mathrm{D}}^{1 / 2}} \frac{\mathrm{d} p_{\mathrm{D}}(\eta)}{\mathrm{d} \eta} \\
& \frac{\mathrm{d} p_{\mathrm{D}}(\eta)}{\mathrm{d} \eta}=-2 t_{\mathrm{D}}^{1 / 2} v_{\mathrm{D}}(\eta)
\end{aligned}
$$

The combination of Eq. (15) with Eq.(14) gives the control equation:

$$
\frac{\mathrm{d} v_{\mathrm{D}}(\eta)}{\mathrm{d} \eta}+\frac{1}{\eta} v_{\mathrm{D}}(\eta)=-2 \eta v_{\mathrm{D}}(\eta)
$$

The general solution to Eq. (16) can be written as follows:

$$
v_{\mathrm{D}}(\eta)=\frac{c_{\mathrm{d}}}{\eta} \exp \left(-\eta^{2}\right)
$$

where $c_{\mathrm{d}}$ is an integral constant.

Substituting Eq. (13) into Eq. (17), we have:

$v_{\mathrm{D}}\left(r_{\mathrm{D}}, t_{\mathrm{D}}\right)=\frac{2 t_{\mathrm{D}}^{1 / 2} c_{\mathrm{d}}}{r_{\mathrm{D}}} \exp \left(-\frac{r_{\mathrm{D}}^{2}}{4 t_{\mathrm{D}}}\right)$

Applying the inner boundary condition $v_{\mathrm{D}}\left(r_{\mathrm{D}}=1, t_{\mathrm{D}}\right)=1$ to Eq. (18) gives:

$$
c_{\mathrm{d}}=\frac{1}{2 t_{\mathrm{D}}^{1 / 2}} \exp \left(\frac{1}{4 t_{\mathrm{D}}}\right)
$$

Thus the dimensionless flow rate can be written as follows:

$$
v_{\mathrm{D}}\left(r_{\mathrm{D}}, t_{\mathrm{D}}\right)=\frac{1}{r_{\mathrm{D}}} \exp \left(\frac{1-r_{\mathrm{D}}^{2}}{4 t_{\mathrm{D}}}\right)
$$

\subsubsection{Non-Darcy flow regime $\left(r_{\mathrm{D}}>r_{\mathrm{cD}}\right)$}

Let

$$
\eta=\frac{r_{\mathrm{D}}}{2 t_{\mathrm{D}}{ }^{1 / 2}}
$$

Substituting Eq. (21) into Eq. (11), we have:

$$
\begin{aligned}
& \frac{\mathrm{d} v_{\mathrm{D}}(\eta)}{\mathrm{d} \eta}+\frac{1}{\eta} v_{\mathrm{D}}(\eta)=\frac{\eta}{t_{\mathrm{D}}^{1 / 2}} \frac{\mathrm{d} p_{\mathrm{D}}(\eta)}{\mathrm{d} \eta} \\
& \frac{\mathrm{d} p_{\mathrm{D}}(\eta)}{\mathrm{d} \eta}=-\frac{2 k v_{\mathrm{w}}^{n-1} c t_{\mathrm{D}}^{1 / 2}}{\mu} v_{\mathrm{D}}^{n}(\eta)
\end{aligned}
$$

The combination of Eq. (23) with Eq. (22) gives the control equation:

$$
\frac{\mathrm{d} v_{\mathrm{D}}(\eta)}{\mathrm{d} \eta}+\frac{1}{\eta} v_{\mathrm{D}}(\eta)=-\frac{2 k v_{\mathrm{w}}^{n-1} c \eta}{\mu} v_{\mathrm{D}}^{n}(\eta)
$$

Eq. (24) is a Bernoulli equation, let

$$
z=v_{\mathrm{D}}^{1-n}(\eta)
$$

Eq. (24) can be reduced as follows:

$\frac{\mathrm{d} z}{\mathrm{~d} \eta}=-\frac{1}{\eta}(1-n) z-\frac{2 k v_{\mathrm{w}}^{n-1} c \eta}{\mu}(1-n)$

The general solution to Eq. (26) can be written as follows:

$$
z=\eta^{n-1}\left[c_{1}+\frac{2 k v_{\mathrm{w}}^{n-1} c}{\mu} \frac{n-1}{3-n} \eta^{3-n}\right]
$$

where $c_{1}$ is an integral constant.

Substituting Eq. (25) into Eq. (27) gives:

$$
v_{\mathrm{D}}(\eta)=\frac{1}{\eta}\left[c_{1}+\frac{2 k v_{\mathrm{w}}^{n-1} c}{\mu} \frac{n-1}{3-n} \eta^{3-n}\right]^{\frac{1}{1-n}}
$$

Using the Boltzmann transformation, Eq. (28) can be expressed as:

$$
v_{\mathrm{D}}\left(r_{\mathrm{D}}, t_{\mathrm{D}}\right)=\frac{2 t_{\mathrm{D}}^{1 / 2}}{r_{\mathrm{D}}}\left[c_{1}-\frac{2 k v_{\mathrm{w}}^{n-1} c}{\mu} \frac{1-n}{3-n}\left(\frac{r_{\mathrm{D}}}{2 t_{\mathrm{D}}^{1 / 2}}\right)^{3-n}\right]^{\frac{1}{1-n}}
$$

Applying the continuity condition $v_{\mathrm{pD}}\left(r_{\mathrm{D}}=r_{\mathrm{CD}}, t_{\mathrm{D}}\right)$ $=v_{\mathrm{dD}}\left(r_{\mathrm{D}}=r_{\mathrm{cD}}, t_{\mathrm{D}}\right)$ to Eq. (29) gives:

$$
c_{1}=\left(\frac{1}{2 t_{\mathrm{D}}^{1 / 2}} \exp \left(\frac{1-r_{\mathrm{cD}}^{2}}{4 t_{\mathrm{D}}}\right)\right)^{1-n}+\frac{2 k v_{\mathrm{w}}^{n-1} c}{\mu} \frac{1-n}{3-n}\left(\frac{r_{\mathrm{cD}}}{2 t_{\mathrm{D}}^{1 / 2}}\right)^{3-n}
$$

Substituting Eq. (30) into Eq. (29) yields:

$$
\begin{aligned}
& v_{\mathrm{D}}\left(r_{\mathrm{D}}, t_{\mathrm{D}}\right)=\frac{1}{r_{\mathrm{D}}}\left[\left(\exp \left(\frac{1-r_{\mathrm{cD}}^{2}}{4 t_{\mathrm{D}}}\right)\right)^{1-n}\right. \\
& \left.+\frac{k v_{\mathrm{w}}^{n-1} c}{2 \mu t_{\mathrm{D}}} \frac{1-n}{3-n}\left(r_{\mathrm{cD}}\right)^{3-n}-\frac{k v_{\mathrm{w}}^{n-1} c}{2 \mu t_{\mathrm{D}}} \frac{1-n}{3-n}\left(r_{\mathrm{D}}\right)^{3-n}\right]^{\frac{1}{1-n}}
\end{aligned}
$$

\subsection{The formation pressure}

\subsubsection{Non-Darcy flow regime $\left(r_{\mathrm{D}}>r_{\mathrm{cD}}\right)$}

Substituting Eq. (31) into the non-Darcy flow equation gives:

$$
\begin{aligned}
& \frac{\partial p_{\mathrm{D}}\left(r_{\mathrm{D}}, t_{\mathrm{D}}\right)}{\partial r_{\mathrm{D}}}=-\frac{k v_{\mathrm{w}}^{n-1} c}{\mu} v_{\mathrm{D}}^{n}\left(r_{\mathrm{D}}, t_{\mathrm{D}}\right) \\
& =-\frac{k v_{\mathrm{w}}^{n-1} c}{\mu} \frac{1}{r_{\mathrm{D}}^{n}} \times\left[\left(\exp \left(\frac{1-r_{\mathrm{cD}}^{2}}{4 t_{\mathrm{D}}}\right)\right)^{1-n}+\frac{k v_{\mathrm{w}}^{n-1} c}{2 \mu t_{\mathrm{D}}} \frac{1-n}{3-n}\left(r_{\mathrm{cD}}\right)^{3-n}\right. \\
& \left.-\frac{k v_{\mathrm{w}}^{n-1} c}{2 \mu t_{\mathrm{D}}} \frac{1-n}{3-n}\left(r_{\mathrm{D}}\right)^{3-n}\right]^{1-n}
\end{aligned}
$$


Integrating Eq. (32) and applying the outer boundary condition $p_{\mathrm{D}}\left(\infty, t_{\mathrm{D}}\right)=0$ gives:

$$
p_{\mathrm{D}}\left(r_{\mathrm{D}}, t_{\mathrm{D}}\right)=\int_{r_{\mathrm{D}}}^{\infty} \frac{k v_{\mathrm{w}}^{n-1} c}{\mu} \frac{1}{r_{\mathrm{D}}^{n}}\left[\begin{array}{l}
\left(\exp \left(\frac{1-r_{\mathrm{cD}}^{2}}{4 t_{\mathrm{D}}}\right)\right)^{1-n}+ \\
\frac{k v_{\mathrm{w}}^{n-1} c}{2 \mu t_{\mathrm{D}}} \frac{1-n}{3-n}\left(r_{\mathrm{cD}}\right)^{3-n}- \\
\frac{k v_{\mathrm{w}}^{n-1} c}{2 \mu t_{\mathrm{D}}} \frac{1-n}{3-n}\left(r_{\mathrm{D}}\right)^{3-n}
\end{array}\right]^{\frac{n}{1-n}} \mathrm{~d} r
$$

\subsubsection{Darcy flow regime $\left(1 \leq r_{\mathrm{D}} \leq r_{\mathrm{cD}}\right)$}

Substituting the Darcy flow equation into Eq. (20) gives:

$$
\frac{\partial p_{\mathrm{D}}\left(r_{\mathrm{D}}, t_{\mathrm{D}}\right)}{\partial r_{\mathrm{D}}}=-v_{\mathrm{D}}\left(r_{\mathrm{D}}, t_{\mathrm{D}}\right)=-\frac{1}{r_{\mathrm{D}}} \exp \left(\frac{1-r_{\mathrm{D}}^{2}}{4 t_{\mathrm{D}}}\right)
$$

In the Darcy flow regime $\left(1 \leq r_{\mathrm{D}} \leq r_{\mathrm{cD}}\right)$, the pressure drawdown is composed of $p_{\mathrm{pD}}$ and $p_{\mathrm{dD}}$, where $p_{\mathrm{pD}}$ is the pressure drawdown in the non-Darcy flow regime; $p_{\mathrm{dD}}$ is the pressure drawdown in the Darcy flow regime.

Then, the dimensionless pressure in the Darcy flow regime:

$$
p_{\mathrm{D}}=p_{\mathrm{dD}}+p_{\mathrm{pD}}
$$

with

$$
\begin{aligned}
& p_{\mathrm{dD}}=\int_{r_{\mathrm{D}}}^{r_{\mathrm{CD}}} \frac{1}{r_{\mathrm{D}}} \exp \left(\frac{1-r_{\mathrm{D}}^{2}}{4 t_{\mathrm{D}}}\right) \mathrm{d} r_{\mathrm{D}} \\
& p_{\mathrm{pD}}=\int_{r_{\mathrm{CD}}}^{\infty} \frac{k v_{\mathrm{w}}^{n-1} c}{\mu} \frac{1}{r_{\mathrm{D}}^{n}}\left[\begin{array}{l}
\left.\frac{k v_{\mathrm{w}}^{n-1} c}{2 \mu t_{\mathrm{D}}} \frac{1-n}{3-n}\left(r_{\mathrm{cD}}\right)^{3-n}-\right)^{1-n}+ \\
\frac{k v_{\mathrm{w}}^{n-1} c}{2 \mu t_{\mathrm{D}}} \frac{1-n}{3-n}\left(r_{\mathrm{D}}\right)^{3-n}
\end{array}\right]^{\frac{n}{1-n}} \mathrm{~d} r_{\mathrm{D}}
\end{aligned}
$$

\subsection{Bottom-hole pressure}

Substituting $r_{\mathrm{D}}=1$ into Eq. (35) gives

$$
\begin{aligned}
p_{\mathrm{wD}}\left(t_{\mathrm{D}}\right)=\int_{1}^{r_{\mathrm{CD}}} \frac{1}{r_{\mathrm{D}}} \exp \left(\frac{1-r_{\mathrm{D}}^{2}}{4 t_{D}}\right) \mathrm{d} r_{\mathrm{D}}+ \\
\int_{r_{\mathrm{cD}}}^{\infty} \frac{k v_{\mathrm{w}}^{n-1} c}{\mu} \frac{1}{r_{\mathrm{D}}^{n}}\left[\begin{array}{l}
\left(\exp \left(\frac{1-r_{\mathrm{cD}}^{2}}{4 t_{\mathrm{D}}}\right)\right)^{1-n}+ \\
\frac{k v_{\mathrm{w}}^{n-1} c}{2 \mu t_{\mathrm{D}}} \frac{1-n}{3-n}\left(r_{\mathrm{cD}}\right)^{3-n}- \\
\frac{k v_{\mathrm{w}}^{n-1} c}{2 \mu t_{\mathrm{D}}} \frac{1-n}{3-n}\left(r_{\mathrm{D}}\right)^{3-n}
\end{array}\right]^{\frac{n}{1-n}} \mathrm{~d} r_{\mathrm{D}}
\end{aligned}
$$

Taking a time step $\Delta t$, so $t_{i \mathrm{D}}=i \Delta t$. If $i=0$, from the initial condition: $p_{\mathrm{wD}}\left(t_{\mathrm{D}}=0\right)=0$; otherwise $t_{i \mathrm{D}}=i \Delta t, i=1,2,3 \cdots$

$$
\begin{aligned}
& p_{\mathrm{wD}}\left(t_{i \mathrm{D}}\right)= \int_{1}^{r_{\mathrm{cD}}} \frac{1}{r_{\mathrm{D}}} \exp \left(\frac{1-r_{\mathrm{D}}^{2}}{4 t_{\mathrm{iD}}}\right) \mathrm{d} r_{\mathrm{D}}+ \\
& {\left[\left(\exp \left(\frac{1-r_{\mathrm{cD}}^{2}}{4 t_{i \mathrm{D}}}\right)\right)^{1-n}+\right.} \\
& \int_{r_{\mathrm{cD}}}^{\infty} \frac{k v_{\mathrm{w}}^{n-1} c}{\mu} \frac{1}{r_{\mathrm{D}}^{n}}\left[\begin{array}{l}
\frac{k v_{\mathrm{w}}^{n-1} c}{2 \mu t_{\mathrm{iD}}} \frac{1-n}{3-n}\left(r_{\mathrm{cD}}\right)^{3-n}- \\
\frac{k v_{\mathrm{w}}^{n-1} c}{2 \mu t_{\mathrm{iD}}} \frac{1-n}{3-n}\left(r_{\mathrm{D}}\right)^{3-n}
\end{array}\right]^{\frac{n}{1-n}} \mathrm{~d} r_{\mathrm{D}}
\end{aligned}
$$

The time derivative of the bottom-hole pressure can be expressed:

$$
\frac{\partial p_{\mathrm{wD}}\left(t_{\mathrm{iD}}\right)}{\partial t_{\mathrm{D}}}=\frac{p_{\mathrm{wD}}\left(t_{(i+1) \mathrm{D}}\right)-p_{\mathrm{wD}}\left(t_{(i-1) \mathrm{D}}\right)}{2 \Delta t}, \quad i=1,2, \cdots
$$

\section{Results and discussion}

Based on the above solutions, the dimensionless bottomhole pressure and its derivative for non-Darcy flow in lowpermeability reservoirs were obtained under different boundary conditions. Fig. 4 presents a graph of the logarithm of the dimensionless bottom-hole pressure versus the logarithm of dimensionless time and its derivative with respect to time for different wellbore storage coefficients $\left(c_{\mathrm{D}}=10^{3}, 10^{4}, 10^{5}\right)$ and different flow regimes $\left(r_{\mathrm{dD}}=20,50\right.$, $100)$. Due to the influence of non-Darcy flow, the derivative curve will be upward sloping, it is a slanted line instead of a 0.5 valued horizontal line.

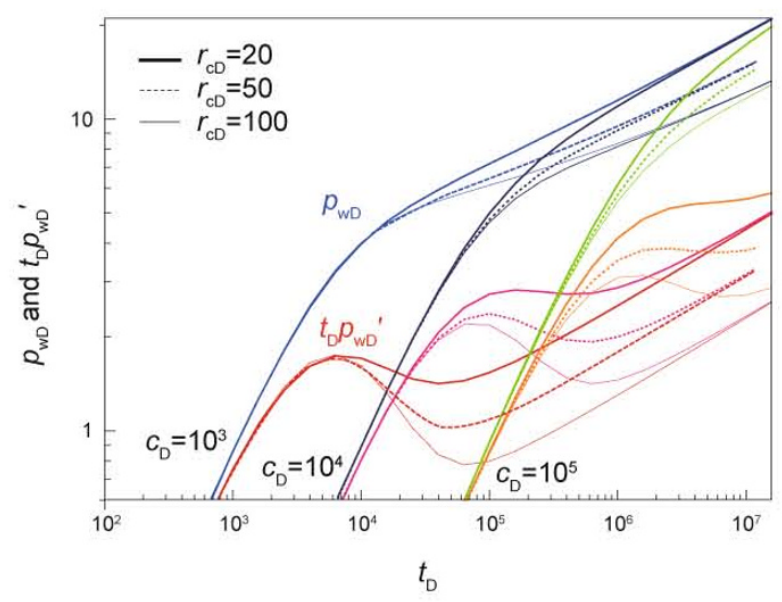

Fig. 4 Dimensionless curves for bottom-hole pressure and its derivative under different wellbore storage coefficients and flow patterns

\subsection{The influence of distribution of reservoir flow patterns}

For reservoirs producing at a given production rate, higher viscosity of the crude oil and lower reservoir permeability will lead to larger zones in which the flow does not obey 
Darcy's law. This means a smaller critical radius. For a specific reservoir, the change of production rate can also result in different distribution of flow patterns. As a consequence, we considered the distribution of Darcy flow and non-Darcy flow; the dimensionless critical radii were 20, 50, 100, 500, and 1,000 . Then we compared the results from the complex flow model with those from the Darcy flow model under different distribution of flow patterns, and the results are shown in Fig. 5. The pressure derivative curve in Fig. 5 shows that the bigger the critical radius $r_{\mathrm{cD}}$ of the complex reservoir is, the later upwards the pressure derivative curve bends. This means non-Darcy flow happens later. Therefore, the flow of fluids from the reservoir is mainly governed by Darcy flow in these wells, under these conditions the pressure derivative curves bend upwards later and their flow coefficients in the well control area are good. The pressure curves in Fig. 5 indicate that for the complex reservoirs the dimensionless pressure obtained from the non-Darcy flow model is larger than that from the Darcy flow model; and the smaller the critical radius $r_{\mathrm{cD}}$ is, the higher the dimensionless pressure is. This means that the larger the area of non-Darcy flow is, the earlier the curve of dimensionless pressure curve will deviate from the Darcy curve.

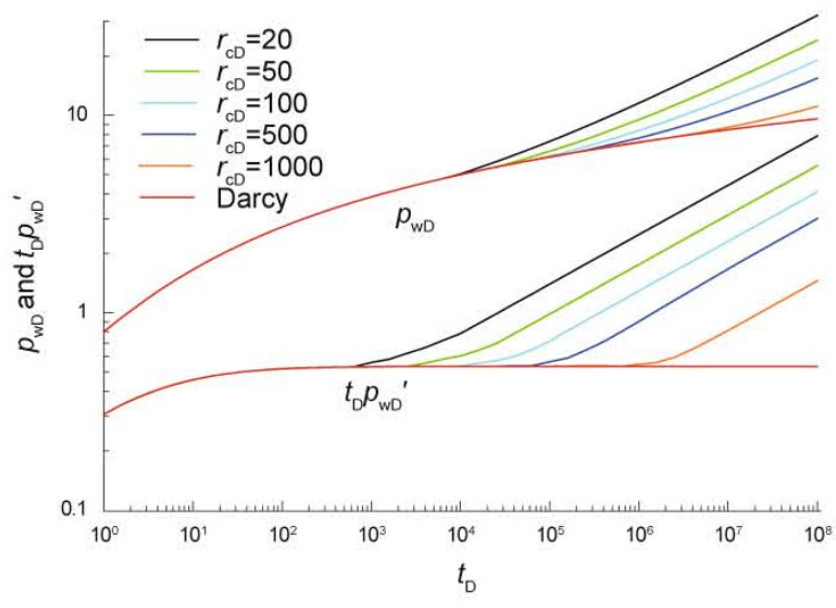

Fig. 5 Dimensionless curves for bottom-hole pressure and its derivative under different distribution of flow patterns

\subsection{The influence of seepage index}

The non-Darcy seepage index obtained from core flow tests was 0.3987 , at which the experimental results had the minimum error. In order to analyze the sensitivity of seepage index, we constructed a log-log plot of dimensionless pressure and pressure derivative versus dimensionless time at different seepage indices, as shown in Fig. 6. The slope of the pressure curve decreases with an increase in the seepage index. When the seepage index is unity, the flow obeys Darcy's law in the whole reservoir and the dimensionless pressure derivative curve is a horizontal line with the value of 0.5 . The dimensionless pressure curve for Darcy flow locates at the bottom, dimensionless pressure increases slowest with time. With the decrease in the seepage index, the slope of the pressure curve increases, and the dimensionless pressure increases more quickly with time. When the seepage index

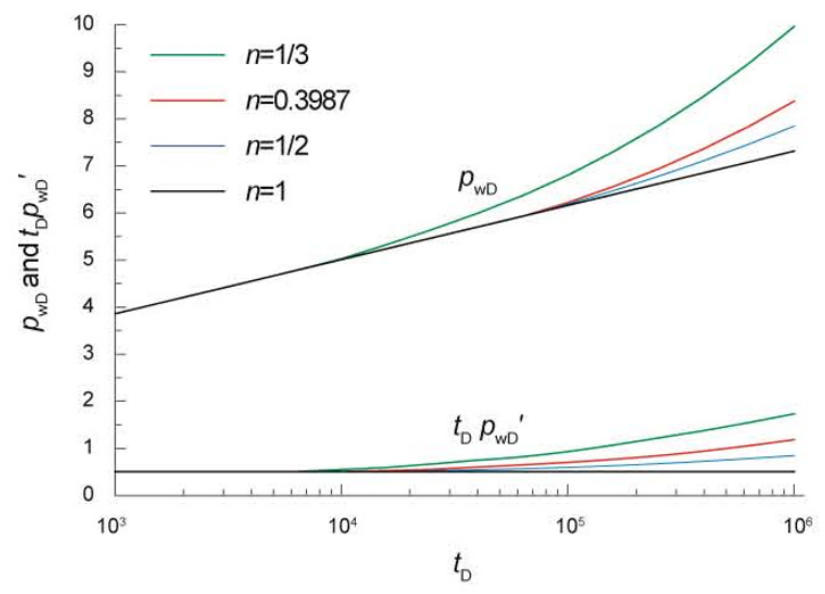

Fig. 6 Dimensionless curves for bottom-hole pressure and its derivative under different seepage indexes

changes from $1 / 2$ to $1 / 3$, the seepage curves (bottom-hole pressure and its derivative) are similar to each other. This indicates that all the pressure derivative curves bend upwards and the slope decreases with an increase in the seepage index.

\section{Conclusions}

This paper analyzed the experimental data with the dimensionless method and established a combined transient flow mathematical model of low-permeability reservoirs. The exact solution to the model was given and the typical curve was discussed.

1) Fluid flow in low-permeability reservoirs: when $R \mathrm{e}<R \mathrm{e}_{\mathrm{c}}, \frac{\Delta p}{\Delta l}=c_{1} v^{0.3987} ;$ while $R \mathrm{e}>R \mathrm{e}_{\mathrm{c}}, v=\frac{k}{\mu} \frac{\Delta p}{\Delta l}$. The critical Reynolds number, $R_{\mathrm{c}}$ is $8.5 \times 10^{-5}$.

2) Characteristics of non-Darcy flow in low-permeability reservoirs: the slope of the dimensionless pressure increases, the pressure derivative curve is not a horizontal line at the value of 0.5 , but a slanted line.

3) With the decrease in non-Darcy seepage index, the slope of the dimensionless pressure curve increases, and the dimensionless pressure increases more quickly with time. When the seepage index varies from $1 / 2$ to $1 / 3$, the distribution of flow patterns are similar to each other. This indicates that the dimensionless curves for the pressure and its derivative slope upwards and the slope decreases with the increase in the seepage index.

\section{Acknowledgements}

This work was supported by the National Natural Science Foundation of China (Grant No.40974055) and the National Key Technology R\&D Program in the 11th Five-Year Plan Period (Grant No. 2008ZX05030-005-03).

\section{References}

Bordier C and Zimmer D. Drainage equations and non-Darcian modelling in coarse porous media or geosynthetic materials. Journal of Hydrology. 2000. (228): 174-187

Cheng S Q, Xu L X and Zhang D C. Type curve matching of well test data for non-Darcy flow at low velocity. Petroleum Exploration and 
Development. 1996. 23(4): 50-53 (in Chinese)

Deng Y E, Huang R Q and Liu C Q. Nonlinear flow law and consolidation in unsaturated low-permeability clays. Journal of Hydrodynamics, Serial A. 2009. 24 (1): 99-105 (in Chinese)

Deng Y E and Liu C Q. A mathematical model of nonlinear flow law in low permeability porous media and its application. Acta Petrolei Sinica. 2001. 22(4): $72-76$ (in Chinese)

Deng Y E, Xie H P, Huang R Q, et al. Law of nonlinear flow in saturated clays and radial consolidation. Applied Mathematics and Mechanics. 2007. 28 (11): 1427-1436

Hou Y M and Tong D K. Nonsteady flow of non-Newtonian power-law fluids of low permeability with moving-boundary in double porous media and fractal reservoir. Engineering Mechanics. 2009. 26(8): 245-250 (in Chinese)

Huang Y Z. Fluid Mechanics in Low-permeability Reservoir. Beijing: Petroleum Industry Press. 1998. 131-135 (in Chinese)

Ikoku C U and Ramey Jr H J. Transient flow of non-Newtonian power law fluids in porous media. SPE Journal. 1979. 44 (3): 164-174

Ikoku C U and Ramey H J. Pressure behavior during polymer flow in petroleum reservoirs. Journal of Energy Resources Technology. 1982. 104: $149-156$

Jiang L Z, Gu J Y and Guo B C. Characteristics and mechanism of low permeability clastic reservoirs in Chinese petroliferous basin. Acta Sedimentologica Sinica. 2004. 22(1): 13-18 (in Chinese)

Li M, Diao N R and Fang Z H. Analysis of seepage flow in a confined aquifer with a standing column well. Journal of Hydrodynamics, Serial B. 2007. 19(1): 84-91

Li Z F, He S L and Men C Q. Study on the non-Darcy percolation rules in the low permeable oilfield. Well Testing. 2005. 14(3): 14-17 (in Chinese)

Liu C Q. Approximation solution of seepage with threshold pressure gradient. Chinese Journal of Geotechnical Engineering. 1982. 4(3): 107-109 (in Chinese)

Liu J J and Liu X G. Study of nonlinear seepage of rock of low permeability. Chinese Journal of Rock Mechanics and Engineering.
2003. 22(4): 556-561 (in Chinese)

Ren X J, Zhang G H and Liao F F. Criterion of starting pressure gradient existence of non-Darcy flowing in low permeability porous media. Journal of Liaoning Technical University (Natural Science). 2009. 28(Suppl): 273-276 (in Chinese)

Song F Q and Liu C Q. Analysis of two-phase fluid flow in low permeability reservoirs with the threshold pressure gradient. Journal of the University of Petroleum, China. 1999. 23(3): 47-50, 56 (in Chinese)

Tong D K and Shi L N. The generalized flow analysis of non-Newtonian visco-elastic fluid flows in porous media. Journal of Hydrodynamics, Serial A. 2004. 19(6): 695-701 (in Chinese)

Tong D K and Wang R H. Analysis of non-Newtonian visco-elastic fluid flows in fractal reservoirs. Science in China, Series G. 2004. (01): 102-109 (in Chinese)

Wen Z, Huang G H and Zhan H B. An analytical solution for nonDarcian flow in a confined aquifer using the power law function. Advances in Water Resources. 2008. (31): 44-55

Xiong W, Shen R and Gao S S. Non-linear flow theory in low permeability reservoir and its preliminary application. Journal of Liaoning Technical University (Natural Science). 2009. 28(Suppl): 58-60 (in Chinese)

Yan Q L, He Q X, Wei L G, et al. A laboratory study of percolation characteristics of single phase flow in low-permeability reservoirs. Journal of Xi' an Shiyou University. 1990. 5(2): 1-6 (in Chinese)

Yang Q L. Nonlinear flow theory in ultra-low permeability reservoirs and its application. Ph.D Thesis. Institute of Porous Flow and Fluid Mechanics of CAS. 2007 (in Chinese)

Yao Y D and Ge J L. Study of the fluid flow in low permeability reservoirs. Petroleum Exploration and Development. 2001. 28(4): 73-75 (in Chinese)

Zhang L J and Yue X A. Mechanism for viscoelastic polymer solution percolating through porous media. Journal of Hydrodynamics, Serial B. 2007.19 (2): $241-248$

(Edited by Sun Yanhua) 